\title{
Pacific
}

Journal of

Mathematics

\section{TOPOLOGY OF POSITIVELY CURVED \\ 8-DIMENSIONAL MANIFOLDS WITH SYMMETRY}

ANAND DESSAI 


\title{
TOPOLOGY OF POSITIVELY CURVED 8-DIMENSIONAL MANIFOLDS WITH SYMMETRY
}

\author{
ANAND DESSAI
}

\begin{abstract}
We show that a simply connected 8-dimensional manifold $M$ of positive sectional curvature and symmetry rank $\geq 2$ resembles a rank-one symmetric space in several ways. For example, the Euler characteristic of $M$ is equal to the Euler characteristic of $S^{8}, \mathbb{H} P^{2}$ or $\mathbb{C} P^{4}$. If $M$ is rationally elliptic, then $M$ is rationally isomorphic to a rank-one symmetric space. For torsionfree manifolds, we derive a much stronger classification. We also study the bordism type of 8-dimensional manifolds of positive sectional curvature and symmetry rank $\geq 2$. As an illustration, we apply our results to various families of 8-manifolds.
\end{abstract}

\section{Introduction}

We study the topology of positively curved 8-dimensional manifolds with symmetry rank $\geq 2$. A Riemannian manifold $M$ is said to have positive curvature if the sectional curvature of all its tangent planes is positive. The symmetry rank of $M$ is defined as the rank of its isometry group. Throughout this paper, all manifolds are assumed to be closed, that is, compact without boundary.

At present only a few manifolds are known to admit a Riemannian metric of positive curvature. Besides the examples of Eschenburg and Bazaikin (which are biquotients of dimension 6,7 or 13), all other simply connected positively curved examples ${ }^{1}$ are homogeneous, that is, they admit a metric of positive curvature with transitive isometry group (the latter were classified by Berger, Wallach, Aloff and Bérard Bergery). In dimension greater than 24 all known examples are symmetric of rank one.

MSC2000: 53C20, 57R19, 57S25.

Keywords: positive curvature, torus actions, Euler characteristic, classification of 8-manifolds. Research partially supported by the German Research Foundation (DFG-Schwerpunktprogramm 1154 "Globale Differentialgeometrie") and the Swiss National Science Foundation (grant number 200021-117701).

${ }^{1}$ Recently, Petersen and Wilhelm [2008], Grove, Verdiani and Ziller [2008], and Dearricott all announced the discovery of new 7-dimensional examples with positive curvature. 
Classifications of various strength have been obtained for positively curved manifolds with large symmetry; see the survey [Wilking 2007, Section 4]. Among the measures of "largeness", we shall focus on the symmetry rank.

Grove and Searle [1994] showed that the symmetry rank of a positively curved simply connected $n$-dimensional manifold $M$ is $\leq[(n+1) / 2]$, and that equality occurs if and only if $M$ is diffeomorphic to a sphere or a complex projective space. Wilking [2003] proved that, if the symmetry rank of $M$ is $\geq n / 4+1$ and if $n \geq 10$, then either $M$ is homeomorphic to a sphere or a quaternionic projective space, or $M$ is homotopically equivalent to a complex projective space (it follows from [Dessai and Wilking 2004] that "homotopically equivalent" can be strengthened to "tangentially equivalent" in this classification). Building on [Wilking 2003], Fang and Rong [2005] showed that, if the symmetry rank is no less than [ $(n-1) / 2]$ and if $n \geq 8$, then $M$ is homeomorphic to a sphere, a quaternionic projective space, or a complex projective space.

In dimension eight, the rank-one symmetric spaces $S^{8}, \mathbb{H} P^{2}$ and $\mathbb{C} P^{4}$ are the only known simply connected positively curved examples. In this dimension, the aforementioned work of Grove and Searle and of Fang and Rong says that a positively curved simply connected manifold $M$ is diffeomorphic to $S^{8}$ or $\mathbb{C} P^{4}$ if the symmetry rank of $M$ is $\geq 4$, and homeomorphic to $S^{8}, \mathbb{H} P^{2}$ or $\mathbb{C} P^{4}$ if the symmetry rank is $\geq 3$.

The main purpose of this paper is to give some information on the topology of positively curved 8-dimensional manifolds with symmetry rank $\geq 2$. Our first result concerns the Euler characteristic:

Theorem 1.1. Let $M$ be a simply connected 8-dimensional manifold. If $M$ admits a metric of positive curvature and symmetry rank $\geq 2$, then the Euler characteristic of $M$ is equal to the Euler characteristic of $S^{8}, \mathbb{H} P^{2}$ or $\mathbb{C} P^{4}$; that is, $\chi(M)=2,3$ or 5 .

This information on the Euler characteristic leads to a rather strong classification if one assumes in addition that the manifold is rationally elliptic. Recall that a closed simply connected manifold $M$ is rationally elliptic if its rational homotopy $\pi_{*}(M) \otimes \mathbb{Q}$ is of finite rank. A conjecture attributed to Bott asserts that any nonnegatively curved manifold is rationally elliptic; see [Grove and Halperin 1982, page 172].

Theorem 1.2. Let $M$ be a simply connected positively curved 8-dimensional manifold of symmetry rank $\geq 2$.

(1) If $M$ is rationally elliptic, $M$ has the rational cohomology ring and rational homotopy type of a rank-one symmetric space, that is, of $S^{8}, \mathbb{H} P^{2}$ or $\mathbb{C} P^{4}$.

(2) If $M$ is rationally elliptic and $H^{*}(M ; \mathbb{Z})$ is torsion-free, $M$ is homeomorphic to $S^{8}$, diffeomorphic to $\mathbb{H} P^{2}$, or tangentially equivalent to $\mathbb{C} P^{4}$. 
If one drops the assumption on rational ellipticity and weakens the assumption on the symmetry rank, one can still prove a bound on the Euler characteristic:

Theorem 1.3. Suppoer $M$ is a simply connected positively curved manifold of even dimension $\leq 8$. Assume $S^{1}$ acts smoothly on $M$. If some $\sigma \in S^{1}$ acts isometrically and nontrivially on $M$, then $\chi(M) \geq 2$.

This fits well with the Hopf conjecture on the positivity of the Euler characteristic of even-dimensional positively curved manifolds.

To put our results in perspective, we briefly recall what is known about positively curved manifolds in low dimensions. Next to surfaces, manifolds of positive curvature are only classified in dimension 3; see [Hamilton 1982] and [Perelman 2002; 2003b; 2003a]. In higher dimensions, the only known obstructions to positive curvature for simply connected manifolds are given by the Betti number theorem of [Gromov 1981] and obstructions to positive scalar curvature (for example, the $\alpha$ invariant of Lichnerowicz and Hitchin, and the obstructions in dimension 4 coming from Seiberg-Witten theory). In particular, the Hopf problem that asks whether $S^{2} \times S^{2}$ admits a metric of positive curvature is still open.

The study of low-dimensional positively curved manifolds with positive symmetry rank began with Hsiang and Kleiner [1989] on 4-dimensional manifolds. Their main result says that the Euler characteristic of a simply connected positively curved 4-dimensional manifold $M$ with positive symmetry rank is $\leq 3$. Using [Freedman 1982], they conclude that $M$ is homeomorphic ${ }^{2}$ to $S^{4}$ or $\mathbb{C} P^{2}$. Rong [2002] showed that a simply connected positively curved 5-dimensional manifold with symmetry rank 2 is diffeomorphic to $S^{5}$. In dimension 6 and 7 there are examples with symmetry rank 2 and 3, respectively, that are not homotopically equivalent to a rank-one symmetric space [Aloff and Wallach 1975; Eschenburg 1982]. This indicates that, in these dimensions, a classification below the maximal symmetry rank is more complicated.

Theorems 1.1 and 1.3 imply that, for many nonnegatively curved manifolds, any metric of positive curvature must be quite nonsymmetric. For example, if $M$ is of even dimension $\leq 8$ and has Euler characteristic $<2$ (for example, if $M$ is a product of two simply connected odd-dimensional spheres, or $M$ is a simply connected Lie group), then it follows from Theorem 1.3 that, for any positively curved metric $g$ on $M$, the only isometry of $(M, g)$ sitting in a compact connected Lie subgroup of the diffeomorphism group is the identity. As a further illustration of our results, we consider the following classes of manifolds:

Product manifolds. Let $M=N_{1} \times N_{2}$ be a simply connected product manifold of dimension 8 (with $\operatorname{dim} N_{i}>0$ ). It is straightforward to see that the Euler characteristic of $M$ is $\neq 2,3$ or 5. By Theorem 1.1, $M$ does not admit a metric of

${ }^{2}$ According to the recent preprint [Kim 2008], $M$ is diffeomorphic to $S^{4}$ or $\mathbb{C} P^{2}$. 
positive curvature with symmetry rank $\geq 2$. In particular, the product of two simply connected nonnegatively curved manifolds $N_{1}$ and $N_{2}$ (for example, $S^{4} \times S^{4}$ ) does not admit a metric of positive curvature and symmetry rank $\geq 2$. It is interesting to compare this with [Hsiang and Kleiner 1989], which implies that $S^{2} \times S^{2}$ does not admit a metric of positive curvature and symmetry rank $\geq 1$.

Connected sum of rank-one symmetric spaces. Cheeger [1973] has shown that $\mathbb{C} P^{4} \sharp \pm \mathbb{C} P^{4}, \mathbb{C} P^{4} \sharp \pm \mathbb{H} P^{2}$ and $\mathbb{H} P^{2} \sharp \pm \mathbb{H} P^{2}$ admit a metric of nonnegative curvature. The Euler characteristic of these manifolds is 8,6 and 4, respectively. By Theorem 1.1, none of them admits a metric of positive curvature and symmetry rank $\geq 2$.

Cohomogeneity-one manifolds. Grove and Ziller [2000] constructed invariant metrics of nonnegative curvature on cohomogeneity-one manifolds with codimensiontwo singular orbits. Using this construction, they exhibited metrics of nonnegative curvature on certain infinite families of simply connected manifolds that fiber over $S^{4}, \mathbb{C} P^{2}, S^{2} \times S^{2}$ or $\mathbb{C} P^{2} \sharp \pm \mathbb{C} P^{2}$ [Grove and Ziller 2000; 2008]; see also the survey [Ziller 2007]. In dimension 8 , the Euler characteristic of all these manifolds turns out to be $\neq 2,3,5$. Again, by Theorem 1.1, none of them admits a metric of positive curvature and symmetry rank $\geq 2$.

Biquotients. Another interesting class of manifolds known to admit metrics of nonnegative curvature are biquotients. A biquotient of a compact Lie group $G$ is the quotient of a homogeneous space $G / H$ by a free action of a subgroup $K$ of $G$, where the $K$-action is induced from the left $G$-action on $G / H$. Note that any homogeneous space can be described as a biquotient by taking one of the factors to be trivial. If $G$ is equipped with a biinvariant metric, the biquotient $M=K \backslash G / H$ inherits a metric of nonnegative curvature, a consequence of O'Neill's formula for Riemannian submersions. As pointed out by Eschenburg [1992], a manifold $M$ is a biquotient if and only if $M$ is the quotient of a compact Lie group $G$ by a free action of a compact Lie group $L$, where the action of $L$ on $G$ is given by a homomorphism $L \rightarrow G \times G$ together with the two-sided action of $G \times G$ on $G$ given by $\left(g_{1}, g_{2}\right)(g):=g_{1} \cdot g \cdot g_{2}^{-1}$.

The topology of biquotients has been investigated in [Eschenburg 1992; Singhof 1993; Kapovitch 2002; Kapovitch and Ziller 2004; Totaro 2002]. Kapovitch and Ziller [2004] have classified biquotients with singly generated rational cohomology. Combining their classification with the first part of Theorem 1.2 gives this:

Corollary 1.4. A simply connected 8-dimensional biquotient of positive curvature and symmetry rank $\geq 2$ is diffeomorphic to $S^{8}, \mathbb{C} P^{4}, \mathbb{H} P^{2}$ or $G_{2} / S O(4)$.

In view of Theorems 1.1 and 1.3, the examples above contain plenty of examples of simply connected nonnegatively curved manifolds with positive Ricci curvature 
for which the metric cannot be deformed to a metric of positive curvature via a symmetry-preserving process such as the Ricci flow.

The paper is structured as follows: In Section 2, we recall basic geometric and topological properties of positively curved manifolds with symmetry. In Section 3, we prove the statements on the Euler characteristic. In Section 4, we prove our classification theorem 1.2 for rationally elliptic manifolds and the corollary 1.4 for biquotients. In the final Section 5, we study the bordism type of positively curved 8-dimensional manifolds with symmetry.

\section{Tools from geometry and topology}

On the topological side, the proofs rely on arguments from equivariant index theory (Theorem 2.4) and the cohomological structure of fixed point sets of smooth actions on cohomology spheres and cohomology projective spaces (Theorem 2.5).

On the geometric side, the proofs rely on the work of Hsiang and Kleiner on positively curved 4-dimensional manifolds with symmetry (Theorem 2.3), the fixed point theorems of Berger, Synge and Weinstein for isometries [Kobayashi 1972, Chapter II, Corollary 5.7; Synge 1936; Weinstein 1968], and the following two properties of totally geodesic submanifolds, which we state for further reference:

Intersection theorem [Frankel 1961]. Let $M$ be a connected positively curved manifold of dimension $n$, and let $N_{1}$ and $N_{2}$ be totally geodesic submanifolds of dimension $n_{1}$ and $n_{2}$, respectively. If $n_{1}+n_{2} \geq n$, then $N_{1}$ and $N_{2}$ intersect.

Here, the dimension of a manifold is defined to be the maximal number occurring as the dimension of a connected component of it. Similarly, the codimension of a submanifold $N$ of a connected manifold $M$ is defined to be the minimal number occurring as the codimension of a connected component of $N$ in $M$.

Building on the intersection theorem, Frankel [1966, page 71] observed that the inclusion of a connected totally geodesic submanifold $N$ is 1-connected if the codimension of $N$ in $M$ is at most half of the dimension of $M$. Using a Morse theory argument, Wilking proved the following far-reaching generalization:

Connectivity theorem [Wilking 2003]. Let $M$ be a connected positively curved manifold, and let $N_{1}$ and $N_{2}$ be connected totally geodesic submanifolds of codimension $k_{1}$ and $k_{2}$, respectively.

(1) Then the inclusion $N_{i} \hookrightarrow M$ is $\left(n-2 k_{i}+1\right)$-connected.

(2) If $k_{1}+k_{2} \leq n$ and $k_{1} \leq k_{2}$, then the intersection $N_{1} \cap N_{2}$ is a totally geodesic submanifold, and the inclusion of $N_{1} \cap N_{2}$ in $N_{2}$ is $\left(n-\left(k_{1}+k_{2}\right)\right)$-connected.

The connectivity theorem leads to strong restrictions on the topology of positively curved manifolds with large symmetry. Wilking [2003] used this property to classify positively curved manifolds of dimension $n \geq 10$ (respectively, $n \geq 6000$ ) 
with symmetry rank $\geq n / 4+1$ (respectively, $\geq n / 6+1$ ). For further reference, we point out the following rather elementary consequences:

Corollary 2.1. Let $M$ be a simply connected positively curved manifold of even dimension $n=2 m \geq 6$.

(1) If $M$ admits a totally geodesic connected submanifold $N$ of codimension 2, then $N$ is simply connected. Moreover, the integral cohomology of $M$ and $N$ is concentrated in even degrees and satisfies $H^{2 i}(M ; \mathbb{Z}) \cong H^{2 j}(N ; \mathbb{Z})$ for all $0<2 i<n$ and $0<2 j<n-2$.

(2) If $M$ admits two different totally geodesic connected submanifolds $N_{1}$ and $N_{2}$ of codimension 2, then $M$ is homeomorphic to $S^{n}$ or homotopy equivalent to $\mathbb{C} P^{m}$.

Proof. Part (1) follows directly from the connectivity theorem; see [Wilking 2003]. For the convenience of the reader, we recall the argument. We begin with a more general discussion:

Let $M$ be an oriented $n$-dimensional connected manifold, and $N \stackrel{i}{\hookrightarrow} M$ an oriented connected submanifold of codimension $k$. Let $u \in H^{k}(M ; \mathbb{Z})$ be the Poincaré dual of the fundamental class of $N$ in $M$. Then, the cup product with $u$ is given by the composition

$$
H^{i}(M ; \mathbb{Z}) \stackrel{i^{*}}{\rightarrow} H^{i}(N ; \mathbb{Z}) \stackrel{\cong}{\longrightarrow} H_{n-k-i}(N ; \mathbb{Z}) \stackrel{i_{*}}{\rightarrow} H_{n-k-i}(M ; \mathbb{Z}) \stackrel{\cong}{\longrightarrow} H^{i+k}(M ; \mathbb{Z})
$$

of maps, where the second and fourth map are the Poincaré isomorphism maps of $N$ and $M$, respectively; see for example [Milnor and Stasheff 1974, page 137]. Now, assume that the inclusion $i: N \hookrightarrow M$ is $(n-k-l)$-connected. Then it is straightforward to check that the homomorphism

$$
\cup u: H^{i}(M ; \mathbb{Z}) \rightarrow H^{i+k}(M ; \mathbb{Z}), \quad x \mapsto x \cup u,
$$

is surjective for $l \leq i<n-k-l$, and injective for $l<i \leq n-k-l$ (for all this, see [Wilking 2003, Lemma 2.2]).

In the first part of the Corollary 2.1, we have $k=2$. By the connectivity theorem, the inclusion $N \hookrightarrow M$ is $(n-3)$-connected, that is, $l=1$. Hence, the map $\cup u$ is surjective for $1 \leq i<n-3$, and injective for $1<i \leq n-3$. Since $M$ is simply connected and $N \hookrightarrow M$ is at least 3-connected, the first part follows.

Next, assume $N_{1}$ and $N_{2}$ are different totally geodesic connected submanifolds of codimension 2. The second part of the connectivity theorem implies that $N:=$ $N_{1} \cap N_{2} \hookrightarrow N_{1}$ is a totally geodesic submanifold, and that the inclusion $N:=$ $N_{1} \cap N_{2} \hookrightarrow N_{1}$ is at least 2-connected.

If the codimension of $N$ in $N_{1}$ is 2 , the map $H^{0}\left(N_{1} ; \mathbb{Z}\right) \rightarrow H^{2}\left(N_{1} ; \mathbb{Z}\right)$ (given by multiplication with the Poincare dual of $N$ in $N_{1}$ for some fixed orientation of $N$ ) is 
surjective by the connectivity theorem. Hence, $b_{2}(M)=b_{2}\left(N_{1}\right) \leq 1$. Using the first part of the Corollary 2.1, we conclude that $M$ is an integral-cohomology sphere if $b_{2}(M)=0$, or an integral-cohomology $\mathbb{C} P^{m}$ if $b_{2}(M)=1$. If $b_{2}(M)=0$, then $M$ is actually homeomorphic to $S^{n}$ by the work of [Smale 1961]; if $b_{2}(M)=1$, then $M$ is homotopy equivalent to $\mathbb{C} P^{m}$ since $M$ is simply connected.

Next, assume the codimension of $N$ in $N_{1}$ is 1 . Using the connectivity theorem, we see that the inclusion $N \hookrightarrow N_{1}$ is $(n-3)$-connected. Arguing along the lines above, it follows that $M$ is homeomorphic to $S^{n}$. This completes the proof of the second part.

Remark 2.2. For 8-dimensional manifolds, one can show that, under the assumptions of Corollary 2.1(2), $M$ is homeomorphic to $S^{8}$ or homeomorphic to $\mathbb{C} P^{4}$. This follows from the Sullivan's classification of homotopy complex projective spaces [1996]; see the argument in [Fang and Rong 2005, page 85].

Another important geometric ingredient in our proofs is the classification (due to Hsiang and Kleiner) up to homeomorphism of positively curved 4-dimensional manifolds with positive symmetry rank.

Theorem 2.3 [Hsiang and Kleiner 1989]. If $M$ is a positively curved simply connected 4-dimensional manifold with positive symmetry rank, the Euler characteristic of $M$ is 2 or 3 , and hence $M$ is homeomorphic to $S^{4}$ or $\mathbb{C} P^{2}$ by Freedman's work.

In particular, $S^{2} \times S^{2}$ does not admit a metric of positive curvature and positive symmetry rank. Note that Theorem 1.1 gives analogous restrictions for the Euler characteristic in dimension 8.

Among the topological tools used in the proofs are the classical Lefschetz fixed point formula for the Euler characteristic, the rigidity of the signature on oriented manifolds with $S^{1}$-action, and its applications to involutions [Hirzebruch 1968], as well as the $\hat{A}$-vanishing theorem of Atiyah and Hirzebruch [1970] for $S^{1}$-actions on Spin-manifolds.

Theorem 2.4. Let $M$ be an oriented manifold with a smooth nontrivial $S^{1}$-action, and $\sigma \in S^{1}$ be the element of order 2. If $M^{S^{1}}$ and $M^{\sigma}$ denote the fixed point manifolds with respect to the $S^{1}$-action and the $\sigma$-action, respectively, then

(1) $\chi(M)=\chi\left(M^{S^{1}}\right)$;

(2) the equivariant signature $\operatorname{sign}_{S^{1}}(M)$ is constant as a character of $S^{1}$;

(3) $\operatorname{sign}(M)=\operatorname{sign}\left(M^{S^{1}}\right)$, where the orientation of each component of the fixed point manifold $M^{S^{1}}$ is chosen to be compatible with the complex structure of its normal bundle (induced by the $S^{1}$-action) and the orientation of $M$; and 
(4) the signature of $M$ is equal to the signature of a transversal self-intersection $M^{\sigma} \circ M^{\sigma}$.

If , in addition, $M$ is a Spin-manifold, then

(5) the Â-genus vanishes and

(6) the connected components of $M^{\sigma}$ are either all of codimension $\equiv 0 \bmod 4$ (even case), or are all of codimension $\equiv 2 \bmod 4$ (odd case).

Proof. Part (1) is just a version of the classical Lefschetz fixed point theorem; see for example [Kobayashi 1972, Theorem 5.5]. We give here a simple argument: For any prime $p$, choose a triangulation of $M$ that is equivariant with respect to the action of $\mathbb{Z} / p \mathbb{Z} \subset S^{1}$ on $M$. Then a counting argument shows that $\chi(M) \equiv$ $\chi\left(M^{\mathbb{Z} / p \mathbb{Z}}\right) \bmod p$. For $p$ large enough, this implies that $\chi(M)=\chi\left(M^{S^{1}}\right)$ (note that the proof also applies to nonorientable manifolds).

Part (2) follows directly from the homotopy invariance of cohomology, or from the Lefschetz fixed point formula of Atiyah, Bott, Segal and Singer (see [Atiyah and Singer 1968, Theorem 6.12, page 582]), as explained for example in [Bott and Taubes 1989, page 142].

For part (3), we consider as a function in $\lambda \in \mathbb{C}$ the $S^{1}$-equivariant signature $\operatorname{sign}_{S^{1}}(M) \in R\left(S^{1}\right) \cong \mathbb{Z}\left[\lambda, \lambda^{-1}\right]$, and compute the limit $\lambda \rightarrow \infty$ using the Lefschetz fixed point formula; details can be found in [Hirzebruch et al. 1992, page 68].

Part (4) is a result of Hirzebruch; see [Hirzebruch 1968; Atiyah and Singer 1968, Proposition 6.15, page 583]. Hirzebruch shows that the signature of a transversal self-intersection $M^{\sigma} \circ M^{\sigma}$ is the equivariant signature $\operatorname{sign}_{S^{1}}(M)$ evaluated at $\sigma$. The claim follows from the rigidity of the signature; see part (2).

Part (5) is the celebrated $\hat{A}$-vanishing theorem of Atiyah and Hirzebruch [1970]. Using the Lefschetz fixed point formula, they show that the $S^{1}$-equivariant $\hat{A}$-genus extends to a holomorphic function on $\mathbb{C}$ that vanishes at infinity. By a classical result of Liouville, this function has to vanish identically.

For a proof part (6), see [Atiyah and Bott 1968, Proposition 8.46, page 487].

We also point out certain properties of smooth actions on cohomology spheres and cohomology projective spaces:

Theorem 2.5. (1) If $M$ is a $\mathbb{Z} / 2 \mathbb{Z}$-cohomology sphere with $a \mathbb{Z} / 2 \mathbb{Z}$-action, the $\mathbb{Z} / 2 \mathbb{Z}$-fixed point manifold is again a $\mathbb{Z} / 2 \mathbb{Z}$-cohomology sphere, or is empty.

(2) If $M$ is an integral-cohomology sphere with an $S^{1}$-action, the $S^{1}$-fixed point manifold is again an integral-cohomology sphere, or is empty.

(3) If $M$ is a $\mathbb{Z} / 2 \mathbb{Z}$-cohomology complex projective space with a $\mathbb{Z} / 2 \mathbb{Z}$-action such that the $\mathbb{Z} / 2 \mathbb{Z}$-action extends to an $S^{1}$-action, then each component of the $\mathbb{Z} / 2 \mathbb{Z}$-fixed point manifold is again a $\mathbb{Z} / 2 \mathbb{Z}$-cohomology complex projective space. 
(4) The action of an involution on a $\mathbb{Z} / 2 \mathbb{Z}$-cohomology $\mathbb{C} P^{2}$ cannot have only isolated fixed points.

Proof. The first two statements are well-known applications of Smith theory; see [Bredon 1972, Chapter III, Theorems 5.1 and 10.2]. The last two statements follow directly from the general theory on fixed point sets of actions on projective spaces; see [Bredon 1972, Chapter VII, Theorems 3.1 and 3.3].

\section{Euler characteristic}

Here we prove the statements on the Euler characteristic given in Section 1.

Theorem 1.3. Let $M$ be a simply connected positively curved manifold of even dimension $\leq 8$. Assume $S^{1}$ acts smoothly on $M$. If some $\sigma \in S^{1}$ acts isometrically and nontrivially on $M$, then $\chi(M) \geq 2$.

Proof. Since $\sigma \in S^{1}$ acts nontrivially, the dimension of $M$ is positive; that is, $\operatorname{dim} M=2,4,6$ or 8 . In dimension $\leq 4$, the theorem is true for purely topological reasons (Poincaré duality). So, assume the dimension of $M$ is 6 or 8 . Note that $M^{\sigma}$ is nonempty [Weinstein 1968] and the connected components of the fixed point manifold $M^{\sigma}$ are totally geodesic submanifolds. Each is of even codimension, since $\sigma$ preserves orientation.

If $M^{\sigma}$ contains a connected component of codimension 2, then, as pointed out in Corollary 2.1, the connectivity theorem implies that all odd Betti numbers of $M$ vanish. Hence, $\chi(M) \geq 2$ by Poincaré duality.

So, assume codim $M^{\sigma}>2$. Note that any connected component $F \subset M^{\sigma}$ is an $S^{1}$-invariant totally geodesic submanifold of even dimension $\leq 4$. If $F$ is not a point, then $F$ inherits positive curvature from $M$. In this case, $F$ or a twofold cover of $F$ is simply connected by [Synge 1936]. Hence the Euler characteristic of any connected component of $M^{\sigma}$ is positive. From the Lefschetz fixed point formula for the Euler characteristic (see Theorem 2.4(1)), we get

$$
\chi(M)=\chi\left(M^{S^{1}}\right)=\chi\left(\left(M^{\sigma}\right)^{S^{1}}\right)=\chi\left(M^{\sigma}\right)=\sum_{F \subset M^{\sigma}} \chi(F) \geq 1 .
$$

Here, equality holds if and only if $M^{\sigma}$ is connected and $\chi\left(M^{\sigma}\right)=1$. If so, $M^{\sigma}$ must have the $\mathbb{Z} / 2 \mathbb{Z}$-cohomology of a point, of $\mathbb{R} P^{2}$, or of $\mathbb{R} P^{4}$. Note that the connected components of $M^{S^{1}}=\left(M^{\sigma}\right)^{S^{1}}$ are orientable submanifolds of even dimension $\leq 4$. This implies that the case $\chi(M)=1$ can only happen if $M^{S^{1}}$ is a point; see [Bredon 1972, Chapter VII, Theorem 3.1].

However, a smooth $S^{1}$-action on a closed orientable manifold $M$ cannot have exactly one fixed point. To show this, consider the Lefschetz fixed point formula [Atiyah and Singer 1968] for the $S^{1}$-equivariant signature $\operatorname{sign}_{S^{1}}(M)$. The local contribution for $\operatorname{sign}_{S^{1}}(M)$ at an isolated $S^{1}$-fixed point extends to a meromorphic 
function on $\mathbb{C}$, which has at least one pole on the unit circle; see for example [Bott and Taubes 1989, page 142]. Since $\operatorname{sign}_{S^{1}}(M)$, being a character of $S^{1}$, has no poles on the unit circle, the $S^{1}$-action cannot have exactly one fixed point (more generally, this is true for any diffeomorphism of order $p^{l}$, with $p$ an odd prime, as shown by [Atiyah and Bott 1968, Theorem 7.1]). Hence, $\chi(M) \geq 2$.

We remark that the proof simplifies drastically if $M$ has positive symmetry rank; see for example [Püttmann and Searle 2002, Theorem 2]. Note that, by the result above, any metric of positive curvature on $S^{3} \times S^{3}, S^{2} \times S^{3} \times S^{3}, S^{3} \times S^{5}$ or $S U$ (3) must be very nonsymmetric.

In the remaining part of this section, we restrict to positively curved simply connected 8-dimensional manifolds with symmetry rank $\geq 2$, and prove the statement on the Euler characteristic given in Theorem 1.1.

Let $T$ be a two-dimensional torus that acts isometrically and effectively on $M$, let $T_{2} \cong \mathbb{Z} / 2 \mathbb{Z} \times \mathbb{Z} / 2 \mathbb{Z}$ denote the 2-torus in $T$, and let $\sigma \in T$ be a nontrivial involution, that is, $\sigma \in T_{2}$ with $\sigma \neq$ id.

By the fixed point theorem of [Weinstein 1968], the fixed point manifold $M^{\sigma}$ is nonempty. Each connected component $F$ of $M^{\sigma}$ is a totally geodesic $T$-invariant submanifold of $M$. Since $\sigma$ preserves orientation, $F$ is of even codimension. By Berger's fixed point theorem (see [Kobayashi 1972, Chapter II, Corollary 5.7]), the torus $T$ acts with fixed point on $F$.

Lemma 3.1. $F$ is orientable. If $\operatorname{dim} F \neq 6$, then $F$ is homeomorphic to $S^{4}, \mathbb{C} P^{2}$, $S^{2}$ or a point.

Proof. If $\operatorname{dim} F=6$, then $F$ is simply connected by the connectivity theorem, and hence orientable.

Next, suppose that $\operatorname{dim} F=4$ and $T$ acts trivially on $F$. In this case, we can choose an $S^{1}$ subgroup of $T$ such that $M^{S^{1}}$ contains a 6-dimensional connected component $Y$, and $F$ is a $T$-fixed point component of $Y$. In this case, by [Grove and Searle 1994, Theorem 1.2] $M, Y$ and $F$ are diffeomorphic to spheres or complex projective spaces,

Suppose that $\operatorname{dim} F=4$ and $T$ acts nontrivially on $F$. In this case, we can find a subgroup $S^{1} \subset T$ and a connected component $Y$ of $M^{S^{1}}$ such that $F \cap Y$ has positive dimension. If $\operatorname{dim} Y=6$, then $M$ is diffeomorphic to $S^{8}$ or $\mathbb{C} P^{4}$; see [Grove and Searle 1994, Theorem 1.2], and $F$ is a $\mathbb{Z} / 2 \mathbb{Z}$-cohomology sphere or a $\mathbb{Z} / 2 \mathbb{Z}$-cohomology complex projective space; see Theorem 2.5. Since the universal cover of $F$ is homeomorphic to $S^{4}$ or $\mathbb{C} P^{2}$ (see Theorem 2.3), we conclude that $F$ is simply connected and homeomorphic to $S^{4}$ or $\mathbb{C} P^{2}$. If $\operatorname{dim} Y=4$, then $Y$ is homeomorphic to $S^{4}$ or $\mathbb{C} P^{2}$ by Theorem 2.3. It follows from Theorem 2.5 that any connected component of $Y \cap F$ is a $\mathbb{Z} / 2 \mathbb{Z}$-cohomology sphere or a $\mathbb{Z} / 2 \mathbb{Z}$ cohomology complex projective space. Since $F \cap Y$ has positive dimension, we 
have $\chi(F) \geq 2$. Applying Theorem 2.3 again, we conclude that $F$ is homeomorphic to $S^{4}$ or $\mathbb{C} P^{2}$.

Finally, assume that $\operatorname{dim} F=2$. We choose an $S^{1}$ subgroup of $T$ that fixes $F$ pointwise. Let $Y$ be the connected component of $M^{S^{1}}$ that contains $F$. If $Y$ is 2-dimensional, then $F=Y$ is orientable of positive curvature and hence diffeomorphic to $S^{2}$. If $Y$ is 6-dimensional, then $M$ is diffeomorphic to $S^{8}$ or $\mathbb{C} P^{4}$ [Grove and Searle 1994, Theorem 1.2], which implies that $F \cong S^{2}$. If $Y$ is 4-dimensional, then we may assume that $T$ acts nontrivially on $Y$ (otherwise, we can replace $Y$ by a 6-dimensional connected component for some other $S^{1}$ subgroup). By Theorem 2.3, $Y$ is homeomorphic to $S^{4}$ or $\mathbb{C} P^{2}$. Using Theorem 2.5, we conclude that $F$ is diffeomorphic to $S^{2}$.

In the proof of Theorem 1.1, we will use the concept of the "type" of an involution at a fixed point component of the $T_{2}$-action. This concept is defined as follows: For each nontrivial involution $\sigma \in T_{2}$ and each connected component $X$ of $M^{T_{2}}$, we consider the dimension of the connected component $F$ of $M^{\sigma}$ containing $X$. For fixed $X$, this gives an unordered triple of dimensions, which we call the type of $X$. Note that $F$ is orientable by Lemma 3.1. Since the action of $T$ is orientation-preserving on $F$, any connected component $X$ of $M^{T_{2}}$ is a totally geodesic $T$-invariant submanifold of even dimension. By Berger's fixed point theorem (see [Kobayashi 1972, Chapter II, Corollary 5.7]), $X^{T}$ is nonempty.

We will use the following information on type, which can be easily verified by considering the isotropy representation at a $T$-fixed point in $X$.

Lemma 3.2. (1) The type of $X$ is $(6,6,4),(6,4,2),(6,2,0),(4,4,4),(4,4,0)$ or $(4,2,2)$.

(2) $X$ is an isolated $T_{2}$-fixed point if and only if $X$ is of type $(6,2,0),(4,4,0)$ or $(4,2,2)$.

Example 3.3. Let $M$ be the quaternionic plane

$$
\mathbb{H} P^{2}=\left\{\left[q_{0}, q_{1}, q_{2}\right] \mid q_{i} \in \mathbb{H},\left(q_{0}, q_{1}, q_{2}\right) \neq 0\right\},
$$

where $\left[q_{0}, q_{1}, q_{2}\right]$ denotes the orbit of $\left(q_{0}, q_{1}, q_{2}\right)$ with respect to the diagonal action of nonzero quaternions on $\mathbb{H}^{3}$ from the right. Consider the action of $T=$ $S^{1} \times S^{1}=\left\{(\lambda, \mu) \mid \lambda, \mu \in S^{1}\right\} \subset \mathbb{C} \times \mathbb{C}$ on $M$ via

$$
(\lambda, \mu)\left(\left[q_{0}, q_{1}, q_{2}\right]\right):=\left[\lambda \cdot \sqrt{\mu} \cdot q_{0}, \sqrt{\mu} \cdot q_{1}, \sqrt{\mu} \cdot q_{2}\right] .
$$

Note that although the square root $\sqrt{\cdot}$ is only well-defined up to sign, the action is independent of this choice. Let $\sigma_{1}$ and $\sigma_{2}$ be the involutions in the first and second $S^{1}$ factor of $T$, and let $\sigma_{3}:=\sigma_{1} \cdot \sigma_{2}$ denote the third nontrivial involution. Then, we 
have the following fixed point manifolds:

$$
\begin{array}{ll}
M^{\sigma_{1}}=\{[1,0,0]\} \cup\left\{\left[0, q_{1}, q_{2}\right] \mid q_{i} \in \mathbb{U},\left(q_{1}, q_{2}\right) \neq 0\right\} \cong p t \cup S^{4}, \\
M^{\sigma_{2}}=\left\{\left[q_{0}, q_{1}, q_{2}\right] \mid q_{i} \in \mathbb{C},\left(q_{0}, q_{1}, q_{2}\right) \neq 0\right\} & \cong \mathbb{C} P^{2}, \\
M^{\sigma_{3}}=\left\{\left[j \cdot q_{0}, q_{1}, q_{2}\right] \mid q_{i} \in \mathbb{C},\left(q_{0}, q_{1}, q_{2}\right) \neq 0\right\} & \cong \mathbb{C} P^{2}, \\
M^{T_{2}}=\{[1,0,0]\} \cup\left\{\left[0, q_{1}, q_{2}\right] \mid q_{i} \in \mathbb{C},\left(q_{1}, q_{2}\right) \neq 0\right\} \cong p t \cup S^{2}
\end{array}
$$

Hence, the type of $X=p t$ is $(4,4,0)$, and the type of $X=S^{2}$ is $(4,4,4)$.

The proof of Theorem 1.1 is based on the next three lemmas.

Lemma 3.4. If $\operatorname{dim} M^{\sigma}=6$ for some involution $\sigma \in T$, then the Euler characteristic of $M$ is 2 or 5 .

Proof. Let $N \subset M^{\sigma}$ be the connected component of dimension 6. Note that all other connected components are isolated $\sigma$-fixed points, by the intersection theorem. In view of Corollary 2.1, the odd Betti numbers of $M$ and $N$ vanish, and the even Betti numbers satisfy $b_{2}(M)=b_{4}(M)=b_{6}(M)=b_{2}(N)=b_{4}(N)$. In particular, we have $\chi(M)-\chi(N)=b_{2}(M)$. By the Lefschetz fixed point formula for the Euler characteristic (see Theorem 2.4(1)), this difference is equal to the number of isolated $\sigma$-fixed points.

Suppose isolated $\sigma$-fixed points do occur (otherwise, $b_{2}(M)=0$ and $\chi(M)=2$ ). Using Lemma 3.2(2), we see that an isolated $\sigma$-fixed point is an isolated $T_{2}$ fixed point, of type $(6,2,0)$ or $(4,4,0)$. If some isolated $\sigma$-fixed point is of type $(6,2,0)$, then $M$ contains a 6-dimensional fixed point manifold different from $N$. In this case, $M$ is homeomorphic to $S^{8}$ or homotopy equivalent to $\mathbb{C} P^{4}$; see Corollary 2.1(2). In particular, $\chi(M)=2$ or 5 .

We now consider the remaining case. Suppose all isolated $\sigma$-fixed points are of type $(4,4,0)$. We fix a nontrivial involution $\sigma_{1} \in T$ different from $\sigma$, and denote by $F_{1}$ the 4-dimensional connected component of $M^{\sigma_{1}}$ (note that $F_{1}$ is unique and has nonempty intersection with $N$, by the intersection theorem). Each isolated $\sigma$-fixed point, being of type $(4,4,0)$, is contained in $F_{1}$, and hence the number $d$ of isolated $\sigma$-fixed points is equal to $\chi\left(F_{1}\right)-\chi\left(F_{1} \cap N\right)$. Since $T$ acts nontrivially on $F_{1}$, Theorem 2.3 tells us that $\chi\left(F_{1}\right) \leq 3$. Using Lemma 3.2, we see that the connected components of $\left(F_{1} \cap N\right)^{T_{2}}$ are necessarily 2-dimensional of type $(6,4,2)$. It follows from Lemma 3.1 that at least one of the connected components of $\left(F_{1} \cap N\right)^{T_{2}}$ is diffeomorphic to $S^{2}$. Hence, $b_{2}(M)=d=\chi\left(F_{1}\right)-\chi\left(F_{1} \cap N\right) \leq 1$, which in turn implies $\chi(M)=2$ or 5 .

Remark 3.5. Under the assumptions of Lemma 3.4, $M$ is homeomorphic to $S^{8}$ or $\mathbb{C} P^{4}$. This follows from the proof above, together with the work of Smale [1961] on the high-dimensional Poincaré conjecture, and Sullivan's classification [1996] of homotopy complex projective spaces. 
Lemma 3.6. If $\operatorname{dim} M^{\sigma}=2$ for some involution $\sigma \in T$, then $\chi(M)=2$.

Proof. We first note that the assumption on $\operatorname{dim} M^{\sigma}$ implies that the signature of $M$ vanishes, by Theorem 2.4(4). Since $\chi(M) \equiv \operatorname{sign}(M) \bmod 2$, the Euler characteristic $\chi(M)$ is even.

By Lemma 3.4, we may assume that the dimension of the fixed point manifold $M^{\tau}$ is $\leq 4$ for every nontrivial involution $\tau$ of $T$. Using Lemma 3.2, we see that every $T_{2}$-fixed point component is an isolated fixed point of type $(4,4,0)$ or $(4,2,2)$.

Let $\sigma_{1}$ and $\sigma_{2}$ denote the nontrivial involutions distinct from $\sigma$. Without loss of generality, we may assume that $\operatorname{dim} M^{\sigma_{1}}=4$. Let $F_{1}$ denote the 4-dimensional connected component of $M^{\sigma_{1}}$. Since $T$ acts nontrivially on $F_{1}$, the universal cover of $F_{1}$ is homeomorphic to $S^{4}$ or $\mathbb{C} P^{2}$ by Theorem 2.3. Since all $T_{2}$-fixed points are isolated, the involution $\sigma$ acts on $F_{1}$ with isolated fixed points. By Theorem 2.5, $F_{1}$ cannot be a cohomology $\mathbb{C} P^{2}$. Hence, $\chi\left(F_{1}\right) \leq 2$.

If $\operatorname{dim} M^{\sigma_{2}}<4$, then any $T_{2}$-fixed point component is contained in $F_{1}$ and hence $\chi(M)=\chi\left(F_{1}\right)=2$ by Theorem 1.3. So assume $M^{\sigma_{2}}$ contains a 4-dimensional connected component $F_{2}$. Arguing as above, we see that $\chi\left(F_{2}\right) \leq 2$. Note that $F_{1}$ and $F_{2}$ intersect by the intersection theorem, and $F_{1} \cap F_{2}$ consists of isolated $\sigma$-fixed points. Hence, $\chi(M)=\chi\left(F_{1}\right)+\chi\left(F_{2}\right)-\chi\left(F_{1} \cap F_{2}\right) \leq 3$. Since $\chi(M)$ is even (as pointed out above) and no less than 2 (by Theorem 1.3), the lemma follows.

Lemma 3.7. If $\operatorname{dim} M^{\sigma}=0$ for some involution $\sigma \in T$, then $\chi(M)=2$.

Proof. The proof is very similar to that of Lemma 3.6. By Theorem 2.4(4), the signature of $M$ vanishes. In particular, $\chi(M)$ is even.

Applying Lemma 3.2, we see that a connected component of $M^{T_{2}}$ is of type $(6,2,0)$ or $(4,4,0)$. If some component has type $(6,2,0)$, then, since $\chi(M)$ is even, the Euler characteristic of $M$ is equal to 2 by Lemma 3.4.

So, assume all components of $M^{T_{2}}$ are of type $(4,4,0)$. Let $\sigma_{1} \in T$ be a nontrivial involution distinct from $\sigma$, and let $F_{1}$ denote the unique 4-dimensional connected component of $M^{\sigma_{1}}$. Note that $M^{T_{2}} \subset F_{1}$. By Theorem 2.3, $\chi\left(F_{1}\right) \leq 3$. Since $\chi(M)$ is even, we get $\chi(M)=\chi\left(M^{T_{2}}\right)=\chi\left(F_{1}\right) \leq 2$. Now, the lemma follows from Theorem 1.3.

Proof of Theorem 1.1. Using the lemmas above, we may assume that $\operatorname{dim} M^{\sigma}=4$ for every nontrivial involution $\sigma \in T$. In view of Lemma 3.2, every connected $T_{2}$ fixed point component $X$ is of type $(4,4,0),(4,2,2)$ or $(4,4,4)$. In the first two cases, $X$ is an isolated fixed point, whereas in the third case an inspection of the isotropy representation shows that $X$ is of dimension two.

Let $\sigma_{i} \in T$ for $i=1,2,3$ denote the nontrivial involutions, and let $F_{i}$ denote the unique 4-dimensional connected component of $M^{\sigma_{i}}$. By the intersection theorem, 
any two of the $F_{i}$ intersect. Since $T$ acts nontrivially on $F_{i}$, we have $\chi\left(F_{i}\right) \leq 3$ by Theorem 2.3. If $\chi\left(F_{i}\right)=3$ for some $i$, that is, if $F_{i}$ is homeomorphic to $\mathbb{C} P^{2}$, then $F_{i}$ contains a $T_{2}$-fixed point component of positive dimension (see Theorem 2.5), which is necessarily of type $(4,4,4)$. Hence, if none of the $T_{2}$-fixed point components is of type $(4,4,4)$, then $\chi\left(F_{i}\right) \leq 2$ for all $i$, and

$$
\chi(M)=\sum_{i} \chi\left(F_{i}\right)-\sum_{i<j} \chi\left(F_{i} \cap F_{j}\right) \leq 3 \cdot 2-3=3 .
$$

Since $\chi(M) \geq 2$ by Theorem 1.3, we are done in this case.

In the other case, the intersection of the $F_{i}$ contains a 2-dimensional $T_{2}$-fixed point component $X$ of type $(4,4,4)$. It follows from Lemma 3.1 and Theorem 2.5 that $X$ is diffeomorphic to $\mathbb{C} P^{1}$. Hence $\chi(M) \leq 3 \cdot 3-2 \cdot 2=5$ with equality holding if and only if each $F_{i}$ is homeomorphic to $\mathbb{C} P^{2}$. Moreover, in the equality case, the $T_{2}$-fixed point components distinct from $X$ are all of type $(4,2,2)$, and for each $\sigma_{i}$ the fixed point manifold $M^{\sigma_{i}}$ is the union of $F_{i}$ and a 2-dimensional sphere.

We claim that $\chi(M) \neq 4$. Suppose to the contrary that $\chi(M)=4$. Then we may assume that at least one of the $F_{i}$ (say, $F_{1}$ ) has Euler characteristic equal to 3 . Now, $\chi(M)=4$ implies that the fixed point manifold $M^{\sigma_{1}}$ is the union of $F_{1}$ and an isolated fixed point $q$ (in fact, arguing as for $X$, we see that any $\sigma_{1}$-fixed point component of positive dimension distinct from $F_{1}$ would be diffeomorphic to $\mathbb{C} P^{1}$, implying that $\chi(M)>4$ ). Note that $q$, being an isolated $\sigma_{1}$-fixed point, must be of type $(4,4,0)$. Hence, $q$ belongs to $F_{2}$ and $F_{3}$. This implies that $\chi\left(F_{2}\right)=\chi\left(F_{3}\right)=3$.

On the other hand, $F_{1}^{T_{2}}$ is the union of $X$ and a point $q^{\prime}$, distinct from $q$, which is of type $(4,4,0)$ or $(4,2,2)$. If $q^{\prime}$ has type $(4,4,0)$, then $\chi\left(F_{2}\right) \geq 4$ or $\chi\left(F_{3}\right) \geq 4$, which contradicts $\chi\left(F_{2}\right)=\chi\left(F_{3}\right)=3$. If $q^{\prime}$ has type $(4,2,2)$, then $\chi\left(M^{\sigma_{2}}\right) \geq 5$ and $\chi\left(M^{\sigma_{3}}\right) \geq 5$, which contradicts $\chi(M)=4$.

Hence, $\chi(M) \neq 4$. Since $\chi(M) \leq 5$ and $\chi(M) \geq 2$ (by Theorem 1.3), we get $\chi(M)=2,3,5$.

Recall that, for an nonorientable even-dimensional manifold of positive curvature, a two-fold cover (the orientation cover) is simply connected [Synge 1936]. Hence, Theorem 1.1 implies this:

Corollary 3.8. Let $M$ be an nonorientable 8-dimensional manifold. If $M$ admits a metric of positive curvature and symmetry rank $\geq 2$, then $\chi(M)=1$.

\section{Rationally elliptic manifolds}

In this section, we apply Theorem 1.1 to rationally elliptic manifolds. Recall that a closed simply connected $n$-dimensional manifold $M$ is rationally elliptic if its rational homotopy $\pi_{*}(M) \otimes \mathbb{Q}$ is of finite rank. Rational ellipticity imposes strong 
topological constraints. For example, Halperin has shown that the Euler characteristic of a rationally elliptic manifold is nonnegative, and that all odd Betti numbers vanish if the Euler characteristic is positive; see [1977, Theorem 1', page 175]. By work of [Friedlander and Halperin 1979, Corollary 1.3], the sum of degrees of generators of $\pi_{2 *}(M) \otimes \mathbb{Q}:=\bigoplus_{i} \pi_{2 i}(M) \otimes \mathbb{Q}$ is $\leq n$,

On the other hand, the class of rationally elliptic manifolds contains some interesting families, for example, Lie groups, homogeneous spaces, biquotients and manifolds of cohomogeneity one. Also, all simply connected manifolds presently known to admit a metric of nonnegative curvature are rationally elliptic.

Using the information on the Euler characteristic given in Theorem 1.1, we obtain the following classification result for rationally elliptic manifolds. I thank Mikiya Masuda for explaining to me the properties of $\mathbb{Z} / 2 \mathbb{Z}$-cohomology $\mathbb{C} P^{4}$ 's with $T$-action that are used in the proof.

Theorem 1.2. Suppose $M$ is a simply connected positively curved 8-dimensional manifold of symmetry rank $\geq 2$.

(1) If $M$ is rationally elliptic, $M$ has the rational cohomology ring and the rational homotopy type of a rank-one symmetric space, that is, of $S^{8}, \mathbb{M} P^{2}$ or $\mathbb{C} P^{4}$.

(2) If $M$ is rationally elliptic and $H^{*}(M ; \mathbb{Z})$ is torsion-free, then $M$ is homeomorphic to $S^{8}$, diffeomorphic to $\mathbb{H} P^{2}$, or tangentially equivalent to $\mathbb{C} P^{4}$.

Proof. (1) We will show that $M$ has the rational cohomology ring of $S^{8}$, $P^{2}$ or $\mathbb{C} P^{4}$. From this, one easily deduces that $M$ is formal. Hence, $M$ has the same rational homotopy type as $S^{8}, \mathbb{H} P^{2}$ or $\mathbb{C} P^{4}$.

By Theorem 1.1, the Euler characteristic of $M$ is 2, 3 or 5 . Since $M$ is rationally elliptic, the rational cohomology ring of $M$ is concentrated in even degrees; see [Halperin 1977, Theorem 1', page 175]. If $\chi(M)=2$ or $\chi(M)=3$, then $M$ has the rational cohomology ring of $S^{8}$ or $\llbracket P^{2}$, respectively; this follows directly from Poincare duality. If $\chi(M)=5$, then the rational cohomology ring of $M$ belongs to one of the following three cases:

(1) $b_{2}(M)=0$ and $b_{4}(M)=3$;

(2) $b_{2}(M)=b_{4}(M)=1$, and $x^{2}=0$ for a generator $x$ of $H^{2}(M ; \mathbb{Q})$;

(3) $b_{2}(M)=b_{4}(M)=1$, and $x^{2} \neq 0$ for a generator $x$ of $H^{2}(M ; \mathbb{Q})$.

According to [Friedlander and Halperin 1979, Corollary 1.3], the sum of degrees of generators of $\pi_{2 *}(M) \otimes \mathbb{Q}$ is $\leq 8$. This excludes the first two cases. In fact, in the first case the minimal model of $M$ must have three generators of degree 4, and in the second case the minimal model of $M$ must have generators of degree 2, 4 and 6. So, only the third case can occur; that is, $b_{2}(M)=b_{4}(M)=1$ and $x^{2} \neq 0$ for a generator $x$ of $H^{2}(M ; \mathbb{Q})$. By Poincaré duality, $M$ has the rational cohomology ring of $\mathbb{C} P^{4}$. 
(2) Now assume that $M$ is rationally elliptic and $H^{*}(M ; \mathbb{Z})$ is torsion-free. If $\chi(M)=2$, then $M$ is rationally a sphere by $(1)$. Since $H^{*}(M ; \mathbb{Z})$ is torsion-free, in this case $M$ is an integral cohomology $S^{8}$. Being simply connected, $M$ is a homotopy sphere and hence homeomorphic to $S^{8}$ by [Smale 1961].

If $\chi(M)=3$, then $M$ is an integral-cohomology $\mathbb{H} P^{2}$ by Poincaré duality. We choose the orientation of $M$ for which the signature of $M$ is one. Note that $M$ is 3-connected, and hence $M$ is a Spin manifold. By the Atiyah-Hirzebruch vanishing theorem (see Theorem 2.4(5)), the $\hat{A}$-genus of $M$ vanishes (this follows also from the result of Lichnerowicz [1963] on the vanishing of the $\hat{A}$-genus for Spin manifolds with positive scalar curvature). Since in dimension eight the space of Pontryagin numbers is spanned by the $\hat{A}$-genus and the signature, the manifolds $M$ and $\mathbb{H} P^{2}$ have the same Pontryagin numbers.

From [Smale 1962, Theorem 6.3], it follows that $M$ admits a Morse function with three critical points. The classification results of Eells and Kuiper [1962, theorem on page 216] for these manifolds imply that the diffeomorphism type of $M$ is determined by its Pontryagin numbers, up to connected sums with homotopy spheres. In particular, $M$ and $\mathbb{H} P^{2}$ are homeomorphic and diffeomorphic, up to connected sums, with a homotopy sphere. Recently, Kramer and Stolz used Kreck's surgery theory to show that the action of the group of homotopy spheres on $\mathbb{H} P^{2}$ via connected sum is trivial; see [2007, Theorem A]. Hence, $M$ and $\mathbb{H} P^{2}$ are diffeomorphic.

Finally, we consider the case when $\chi(M)=5$. From (1), we know already that $M$ is a rational cohomology $\mathbb{C} P^{4}$. Below, we will show that $M$ is in fact an integral cohomology $\mathbb{C} P^{4}$. Assuming this for the moment, we now prove that $M$ is tangentially equivalent to $\mathbb{C} P^{4}$; that is, there exists a homotopy equivalence $f: M \rightarrow \mathbb{C} P^{4}$ such that $f^{*}\left(T \mathbb{C} P^{4}\right)$ and $T M$ are stably isomorphic.

We first note that $M$ and $\mathbb{C} P^{4}$ are homotopy equivalent since $M$ is an integral cohomology $\mathbb{C} P^{4}$ and simply connected. In the early 1970s, Petrie conjectured that a smooth $S^{1}$-manifold $N$ that is homotopy equivalent to $\mathbb{C} P^{n}$ has the same Pontryagin classes as $\mathbb{C} P^{n}$; that is, the total Pontryagin class $p\left(\mathbb{C} P^{n}\right)$ is mapped to $p(N)$ under a homotopy equivalence $N \rightarrow \mathbb{C} P^{n}$. Petrie's conjecture holds for $n=4$ [James 1985]. Hence, the homotopy equivalence $f: M \rightarrow \mathbb{C} P^{4}$ maps the Pontryagin classes of $\mathbb{C} P^{4}$ to the Pontryagin classes of $M$. In this situation $M$ and $\mathbb{C} P^{4}$ are tangentially equivalent [Petrie 1973, page 140]. We sketch the argument: Since $H^{*}(M ; \mathbb{Z})$ is torsion-free, the condition on the Pontryagin classes implies that the complexified vector bundles $T M \otimes \mathbb{C}$ and $f^{*}\left(T \mathbb{C} P^{4}\right) \otimes \mathbb{C}$ agree in complex $K$-theory. When $M$ is a homotopy complex projective space of complex dimension $\not \equiv 1 \bmod 4$, the complexification map $K O(M) \rightarrow K(M)$ is injective. Hence, the real vector bundles $T M$ and $f\left(T \mathbb{C} P^{4}\right)$ are stably isomorphic (in fact, they are isomorphic, since they have up to sign the same Euler class). 
To complete the proof, we need to show that $M$ is an integral cohomology $\mathbb{C} P^{4}$. We choose the orientation of $M$ for which the signature of $M$ is 1 . Since $H^{*}(M ; \mathbb{Z})$ is torsion-free, it follows from Poincare duality that $M$ is a twisted $\mathbb{C} P^{4}$; that is, there are generators $x_{2 i} \in H^{2 i}(M ; \mathbb{Z})$ for $i=1,2,3,4$, and an integer $m>0$, such that $x_{8}$ is the preferred generator with respect to the chosen orientation, and

$$
x_{2} \cdot x_{6}=x_{4}^{2}=x_{8}, \quad x_{2}^{2}=m \cdot x_{4}, \quad x_{2} \cdot x_{4}=m \cdot x_{6} .
$$

Let $T$ denote the 2-dimensional torus that acts isometrically and effectively on $M$, let $T_{2} \cong \mathbb{Z} / 2 \mathbb{Z} \times \mathbb{Z} / 2 \mathbb{Z}$ denote the 2-torus in $T$, and let $\sigma \in T_{2}$ be a nontrivial involution. By Theorem 2.4(4), the codimension of $M^{\sigma}$ is 2 or 4. If the codimension of $M^{\sigma}$ is 2 , then $M$ is an integral cohomology $\mathbb{C} P^{4}$; that is, $m=1$. This follows directly from the proof of Corollary 2.1.

So, we are left with the case when $\operatorname{dim} M^{\sigma}=4$ for every nontrivial involution $\sigma \in T$. Let $\sigma_{i} \in T$ for $i=1,2,3$ denote the nontrivial involutions. From the discussion in the previous section (see the proof of Theorem 1.1), we recall the following facts: For each $\sigma_{i}$, the fixed point manifold $M^{\sigma_{i}}$ is the union of a 4dimensional connected component $F_{i}$ and a 2-dimensional sphere $S_{i}^{2}$. Moreover, $F_{i}$ is homeomorphic to $\mathbb{C} P^{2}$, the three $F_{i}$ intersect in a 2-dimensional $T_{2}$-fixed point component $X$ of type $(4,4,4)$, and $X$ is diffeomorphic to $\mathbb{C} P^{1}$. We choose the orientation of $F_{i}$ for which the signature of $F_{i}$ is 1 .

Therefore the normal bundle of $X$ in $M$ is isomorphic as a real vector bundle to three copies of the Hopf bundle. In particular, the normal bundle is not spin and the restriction of the second Stiefel-Whitney class of $M$ to $X$ is nonzero. This shows that the restriction homomorphism $f_{i}^{*}: H^{2}(M ; \mathbb{Z}) \rightarrow H^{2}\left(F_{i} ; \mathbb{Z}\right)$ induced by the inclusion $f_{i}: F_{i} \hookrightarrow M$ maps $x_{2}$ to an odd multiple of a generator $x$ of $H^{2}\left(F_{i} ; \mathbb{Z}\right) \cong$ $H^{2}\left(\mathbb{C} P^{2} ; \mathbb{Z}\right)$; that is, $f_{i}^{*}\left(x_{2}\right)=a \cdot x$ with $a$ odd. It follows from the Lefschetz fixed point formula for the equivariant signature (see [Atiyah and Singer 1968]) and Theorem 2.4(4) that the Euler class $e\left(v_{i}\right)$ of the normal bundle $v_{i}$ of $F_{i} \hookrightarrow M$ is equal to the preferred generator $x^{2} \in H^{4}\left(F_{i} ; \mathbb{Z}\right)$. Hence, $f_{i}{ }^{*}\left(x_{4}\right)=e\left(v_{i}\right)=x^{2}$. By restricting the identity $x_{2}^{2}=m \cdot x_{4}$ to $F_{i}$, we see that $m=a^{2}$ is an odd square. In particular, $M$ is a $\mathbb{Z} / 2 \mathbb{Z}$-cohomology $\mathbb{C} P^{4}$.

Next, we recall from the proof of Theorem 1.1 that $M^{T_{2}}$ is the union of $X$ and three points $p_{i}$ for $i=1,2,3$, with $p_{i} \in F_{i}$. We fix a lift $\xi \in H_{T}^{2}(M ; \mathbb{Z})$ of $x_{2}$ and denote by $w_{i}$ the restriction of $\xi$ to $p_{i}$. By the structure theorem for rational cohomology complex projective spaces [Hsiang 1975, Theorem VI.I, page 106], the kernel of the restriction homomorphism $H_{T}^{*}(M ; \mathbb{Q}) \rightarrow H_{T}^{*}\left(p_{i} ; \mathbb{Q}\right)$ is generated by $\left(\xi-w_{i}\right)$.

The following argument, due to Masuda, shows that $m$ is equal to 1 . Let $f_{i !}$ : $H_{T}^{*}\left(F_{i} ; \mathbb{Z}\right) \rightarrow H_{T}^{*+4}(M ; \mathbb{Z})$ denote the equivariant Gysin map (or push-forward) 
induced by $f_{i}: F_{i} \hookrightarrow M$. For properties of the Gysin map, see for example [Masuda 1981, pages 132-133].

Claim 1: $f_{i !}(1)=\left(1 / a^{2}\right)\left(\xi-w_{j}\right) \cdot\left(\xi-w_{k}\right)$, where $\{i, j, k\}=\{1,2,3\}$.

Proof. Since $p_{j}$ and $p_{k}$ are not in $F_{i}$, the restriction of $f_{i !}(1)$ to each of these points must vanish. Hence, $f_{i !}(1)$ is divisible by $\left(\xi-w_{j}\right) \cdot\left(\xi-w_{k}\right)$. Comparing degrees, we find that $f_{i !}(1)=c \cdot\left(\xi-w_{j}\right) \cdot\left(\xi-w_{k}\right)$ for some rational constant $c$. By restricting this identity to ordinary cohomology, we obtain $x_{4}=c \cdot x_{2}^{2}$, and hence $c=1 / m=1 / a^{2}$.

Claim 2: $w_{i}-w_{j}$ is divisible by $a^{2}$ in $H^{2}(B T ; \mathbb{Z})$.

Proof. From the first claim, we deduce that

$$
f_{i !}(1)-f_{j !}(1)=\frac{1}{a^{2}}\left(\left(w_{i}-w_{j}\right) \cdot \xi-\left(w_{i}-w_{j}\right) \cdot w_{k}\right) .
$$

Since $(1, \xi)$ is part of a basis for the free $H^{*}(B T ; \mathbb{Z})$-module $H_{T}^{*}(M ; \mathbb{Z})$, it follows that $\left(w_{i}-w_{j}\right)$ is divisible by $a^{2}$.

Recall that any two $T$-fixed points $p_{i}$ and $p_{j}$ with $i \neq j$ are contained in a $T$-invariant 2-dimensional sphere $S_{k}^{2}$ that is fixed pointwise by the involution $\sigma_{k}$ (where $\{i, j, k\}=\{1,2,3\})$. Consider the $T$-action on $S_{k}^{2}$, and let $m_{i j} \in H^{2}(B T ; \mathbb{Z})$ denote the weight of the tangential $T$-representation at $p_{i}$. Note that $m_{i j}$ is only defined up to sign and that $m_{i j}= \pm m_{j i}$ (here and in the following the notation $\alpha= \pm \beta$ is a shortcut for $\alpha=\beta$ or $\alpha=-\beta$ ).

Claim 3: $\pm a \cdot m_{i j}=w_{i}-w_{j}$, and $m_{i j}$ is divisible by $a$.

Proof. First, note that the normal bundle of $F_{i}$ in $M$ restricted to $p_{i}$ has weights $\pm m_{i j}$ and $\pm m_{i k}$, where $\{i, j, k\}=\{1,2,3\}$. Hence, by restricting the identity given in the first claim to the $p_{i}$, we obtain the following identities in the polynomial ring $H^{*}(B T ; \mathbb{Z})$ :

$$
\begin{aligned}
& \pm a^{2} \cdot m_{12} \cdot m_{13}=\left(w_{1}-w_{2}\right) \cdot\left(w_{1}-w_{3}\right) \\
& \pm a^{2} \cdot m_{23} \cdot m_{21}=\left(w_{2}-w_{3}\right) \cdot\left(w_{2}-w_{1}\right) \\
& \pm a^{2} \cdot m_{31} \cdot m_{32}=\left(w_{3}-w_{1}\right) \cdot\left(w_{3}-w_{2}\right)
\end{aligned}
$$

Since $m_{i j}$ and $m_{j i}$ agree up to sign, $\pm a \cdot m_{i j}=w_{i}-w_{j}$. Using Claim 2, $m_{i j}$ is divisible by $a$.

Suppose $X \subset M^{T}$. We can choose a subgroup $S^{1}$ of $T$ such that $F_{1}$ is fixed pointwise by $S^{1}$. By Claim 3, the subgroup $\mathbb{Z} / a \mathbb{Z}$ of $S^{1}$ acts trivially on $M$. Since the $T$-action is effective, we get $a= \pm 1$.

If $X \not \subset M^{T}$, then $M^{T}$ consists of five isolated points $\left\{p, p^{\prime}, p_{1}, p_{2}, p_{3}\right\}$, where $p, p^{\prime} \in X$ and $p_{i} \in F_{i}$. Recall that $F_{i}$ is homeomorphic to $\mathbb{C} P^{2}$. In particular, there is a unique $T$-invariant 2-dimensional sphere in $F_{i}$ that contains $p$ and $p_{i}$. 
Consider the $T$-action on this sphere and let $m_{i} \in H^{2}(B T ; \mathbb{Z})$ denote the weight of the tangential $T$-representation at $p_{i}$. Similarly, let $m_{i}^{\prime} \in H^{2}(B T ; \mathbb{Z})$ denote the weight of the tangential $T$-representation that corresponds to $p^{\prime}$ and $p_{i}$. Note that $m_{i}$ and $m_{i}^{\prime}$ are only defined up to sign.

Let $w$ and $w^{\prime}$ denote the restriction of $\xi$ to $p$ and $p^{\prime}$, respectively. Since any torus action on a homotopy $\mathbb{C} P^{2}$ is of linear type and $x_{2}$ restricted to $F_{i}$ is equal to $a$ times a generator of $H^{2}\left(F_{i} ; \mathbb{Z}\right)$, we get

$$
\pm a \cdot m_{i}=w_{i}-w \quad \text { and } \quad \pm a \cdot m_{i}^{\prime}=w_{i}-w^{\prime} .
$$

Now consider the circle subgroup $S \hookrightarrow T$ defined by $w=w^{\prime}$. Since $T$ acts linearly on $F_{i}$, the fixed point set $F_{i}^{S}$ is the union of $X$ and $p_{i}$.

For $u \in H^{*}(B T ; \mathbb{Z})$, let $\bar{u}$ denote the restriction of $u \in H^{*}(B T ; \mathbb{Z})$ to $H^{*}(B S ; \mathbb{Z})$. Since $\bar{w}=\bar{w}^{\prime}$, it follows from equations (1) that $\bar{m}_{i}$ and $\bar{m}_{i}^{\prime}$ agree up to sign. Since $T$ acts effectively on $M$ and the weights $m_{i j}$ are divisible by $a$ (see Claim 3), $\bar{m}_{i}$ and $\bar{m}_{i}^{\prime}$ are both coprime to $a$.

Suppose $m=a^{2}$ is not equal to 1 . Consider the action of $\mathbb{Z} / a \mathbb{Z} \subset S$. Since $\bar{m}_{i j}$ is divisible by $a$, and $\bar{m}_{i}$ and $\bar{m}_{i}^{\prime}$ are both coprime to $a$, the connected $\mathbb{Z} / a \mathbb{Z}$ fixed point component $Z$ that contains $p_{1}$ contains both $p_{2}$ and $p_{3}$ but does not contain $X$. Hence, the $S$-equivariant Gysin map $f_{!}: H_{S}^{*}(Z ; \mathbb{Z}) \rightarrow H_{S}^{*+4}(M ; \mathbb{Z})$ induced by the inclusion $f: Z \hookrightarrow M$ vanishes after restricting to $X$. Applying the structure theorem for rational cohomology complex projective spaces [Hsiang 1975, Theorem VI.I, page 106] to $M$ and $Z$, we find that $f_{!}(1)$ is divisible by $(\bar{\xi}-\bar{w})^{2}$. Comparing degrees, we see that there is a rational constant $C$ such that $f_{!}(1)=C \cdot(\bar{\xi}-\bar{w})^{2}$. By restricting this identity to the $T$-fixed point $p_{i}$, we obtain $\pm \bar{m}_{i} \cdot \bar{m}_{i}^{\prime}=C \cdot\left(\bar{w}_{i}-\bar{w}\right)^{2}$. Using (1), we get $C= \pm 1 / a^{2}$. Hence $\left(1 / a^{2}\right) \cdot(\bar{\xi}-\bar{w})^{2} \in$ $H_{S}^{4}(M ; \mathbb{Z})$. Recall from Claim 1 that $\left(1 / a^{2}\right)\left(\bar{\xi}-\bar{w}_{1}\right) \cdot\left(\bar{\xi}-\bar{w}_{2}\right)$ is also in $H_{S}^{4}(M ; \mathbb{Z})$. Taking the difference of these two elements, we obtain

$$
\frac{1}{a^{2}}\left(\left(\bar{w}_{2}-\bar{w}_{1}+2 \cdot\left(\bar{w}_{1}-\bar{w}\right)\right) \cdot \bar{\xi}+\left(\bar{w}^{2}-\bar{w}_{1} \cdot \bar{w}_{2}\right)\right) \in H_{S}^{4}(M ; \mathbb{Z}) .
$$

Since $(1, \bar{\xi})$ is part of a basis of the free $H^{*}(B S ; \mathbb{Z})$-module $H_{S}^{*}(M ; \mathbb{Z})$, it follows that $\left(\bar{w}_{2}-\bar{w}_{1}+2 \cdot\left(\bar{w}_{1}-\bar{w}\right)\right)$ is divisible by $a^{2}$. Now $\left(\bar{w}_{2}-\bar{w}_{1}\right)$ is divisible by $a^{2}$ by Claim 3, and $a$ is odd. Hence $\left(\bar{w}_{1}-\bar{w}\right)$ is divisible by $a^{2}$. Using (1), we deduce that $a$ divides $\bar{m}_{i}$. This contradicts $a^{2} \neq 1$ since $\bar{m}_{i}$ is coprime to $a$. Hence $m=a^{2}$ is equal to 1 .

We close this section with an application to biquotients. Recall that any biquotient of a compact connected Lie group $G$ is rationally elliptic and comes with a metric of nonnegative curvature induced from a biinvariant metric on $G$.

Corollary 4.1. A simply connected 8-dimensional biquotient of positive curvature and symmetry rank $\geq 2$ is diffeomorphic to $S^{8}, \mathbb{C} P^{4}, \mathbb{H} P^{2}$ or $G_{2} / \mathrm{SO}(4)$. 
Proof. According to Theorem 1.2, a simply connected positively curved 8-dimensional biquotient with symmetry rank $\geq 2$ is rationally singly generated. Rationally singly generated biquotients where classified by Kapovitch and Ziller in [2004, Theorem A]. In dimension 8, these are the homogeneous spaces given.

Remark 4.2. From the classification of homogeneous positively curved manifolds, it follows that $G_{2} / \mathrm{SO}(4)$ does not admit a homogeneous metric of positive curvature. We do not know whether $G_{2} / \mathrm{SO}(4)$ admits a positively curved metric with symmetry rank two.

\section{Bordism type}

In this section, we consider the bordism type of closed simply connected Riemannian 8-manifolds with positive curvature. We determine the Spin-bordism type and comment on the oriented bordism type for manifolds with symmetry rank $\geq 2$.

In dimension eight, the Spin-bordism group $\Omega_{8}^{\text {Spin }}$ is isomorphic to $\mathbb{Z} \oplus \mathbb{Z}$, and the Spin-bordism type is detected by Pontryagin numbers; see [Milnor 1963, page 201]. Since in this dimension the Pontryagin numbers are uniquely determined by the $\hat{A}$-genus and the signature, it suffices to compute these numerical invariants.

Proposition 5.1. Let $M$ be an 8-dimensional Spin-manifold. If $M$ admits a metric of positive curvature and symmetry rank $\geq 2$, then $\chi(M)=2$ or 3 , and $M$ is Spinbordant to $S^{8}$ or $\pm \llbracket P^{2}$.

Proof. By the Atiyah-Hirzebruch vanishing theorem (see Theorem 2.4(5)) or alternatively by the theorem of Lichnerowicz [1963], the $\hat{A}$-genus of $M$ vanishes. Hence, it suffices to show that the signature of $M$ is equal to the signature of $S^{8}$ or $\pm \mathbb{P} P^{2}$; that is, we want to show that $|\operatorname{sign}(M)| \leq 1$. If some isometry in $T$ acts with a fixed point component of codimension 2, this follows directly from Corollary 2.1 and Theorem 1.1 (in fact $M$ is bordant to $S^{8}$ since an integral-cohomology $\mathbb{C} P^{4}$ is never spin). So assume that for any $\tau \in T$ the fixed point manifold

$M^{\tau}$ has no fixed point component of codimension 2 .

Recall from Theorem 1.1 that $\chi(M)=2,3$ or 5 . If $\chi(M)=2, \operatorname{sign}(M)=0$. To see this consider a subgroup $S^{1} \subset T$ of positive fixed point dimension (that is, $\operatorname{dim} M^{S^{1}}>0$ ). By condition (*), any connected component of $M^{S^{1}}$ is of dimension no more than 4. It follows from Theorem 2.4(1) that $M^{S^{1}}$ is $S^{2}$ or an integral cohomology $S^{4}$. Since the signature of $M$ is the sum of the signatures of the connected components of $M^{S^{1}}$, the signature of $M$ vanishes; see Theorem 2.4(3).

If $\chi(M)=3$, then $|\operatorname{sign}(M)|=1$. The reasoning is similar to the one above. Choose an $S^{1}$ subgroup of $T$ such that the fixed point manifold $M^{S^{1}}$ has a connected 
component $F$ of dimension 2 or 4 . Any such $F$ is simply connected by [Synge 1936] and satisfies $|\operatorname{sign}(F)| \leq \chi(F)-2$. Since $\chi(M)=\chi\left(M^{S^{1}}\right)=3$ and the signature of $M$ is the sum of the signatures of the connected components of $M^{S^{1}}$ (taken with the appropriate orientation), we get $|\operatorname{sign}(M)|=1$.

Finally, we claim that the case $\chi(M)=5$ cannot occur. First note that in this case the signature of $M$ is odd $\operatorname{since} \operatorname{sign}(M) \equiv \chi(M) \bmod 2$. Let $\sigma_{i}$ for $i \in\{1,2,3\}$ denote the three nontrivial involutions in $T$. It follows from condition $(*)$ and Theorem 2.4(4) that $M^{\sigma_{i}}$ contains a 4-dimensional connected component $F_{i}$ (which is unique by the intersection theorem). By Lemma 3.1, $F_{i}$ is homeomorphic to $S^{4}$ or $\mathbb{C} P^{2}$. Since $M$ is spin, the action of $\sigma_{i}$ must be even; see Theorem 2.4(6). Hence $M^{\sigma_{i}}$ is the union of $F_{i}$ and isolated $\sigma_{i}$-fixed points. Using Lemma 3.2, we see that each connected component of $M^{T_{2}}$ has type $(4,4,4)$ or $(4,4,0)$. In particular, any $T_{2}$-fixed point component is contained in some $F_{i}$.

To derive a contradiction, we will compute the Euler characteristic. Consider the case when for one of the $F_{i}$, say $F_{1}$, the Euler characteristic is equal to 3 and hence $F_{i}$ is homeomorphic to $\mathbb{C} P^{2}$. Since $\sigma_{2}$ acts nontrivially on $F_{1}$, we get $F_{1}^{\sigma_{2}}=F_{1}^{\sigma_{3}}=S^{2} \cup\{p t\}$, where $S^{2}$ and $p t$ are connected components of $M^{T_{2}}$ of type $(4,4,4)$ and $(4,4,0)$, respectively. Hence, one of the other $F_{i}$, say $F_{2}$, contains $S^{2} \cup\{p t\}$. This leads to the contradiction

$$
5=\chi(M)=\chi\left(M^{T_{2}}\right)=\chi\left(F_{2}\right)+\chi\left(F_{3}\right)-\chi\left(F_{2} \cap F_{3}\right) \leq 3+3-2=4 .
$$

So, assume $\chi\left(F_{i}\right)=2$ for all $i$. Note that $M^{T_{2}}$ cannot contain a connected component of type $(4,4,4)$ since otherwise $\chi(M)=2$ by a computation similar to the one above. Hence, each connected component of $M^{T_{2}}$ is of type $(4,4,0)$. In particular, the $F_{i}$ intersect pairwise in different points, which gives the contradiction

$$
5=\chi(M)=\sum_{i} \chi\left(F_{i}\right)-\sum_{i<j} \chi\left(F_{i} \cap F_{j}\right)+\chi\left(F_{1} \cap F_{2} \cap F_{3}\right) \leq 6-3=3 .
$$

In conclusion, we have shown that $\chi(M) \neq 5$, which completes the proof of the theorem.

A more natural and apparently more difficult problem is to understand the oriented bordism type of an 8-dimensional positively curved manifold $M$ with symmetry rank $\geq 2$.

Wall [1960] has shown that the only torsion in the oriented bordism ring $\Omega_{*}^{\mathrm{SO}}$ is 2-torsion, and that the oriented bordism type of a manifold is determined by Pontryagin and Stiefel-Whitney numbers. In dimension eight, $\Omega_{8}^{S O}$ is isomorphic to $\mathbb{Z} \oplus \mathbb{Z}$. Hence, in this dimension the oriented bordism type of an oriented manifold is determined by its Pontryagin numbers.

In contrast to the case of Spin-manifolds, the $\hat{A}$-genus does not have to vanish on positively curved 8-dimensional oriented manifolds with symmetry (consider for 
example $\mathbb{C} P^{4}$ ). This makes the problem of determining the oriented bordism type more difficult. One way to attack this problem is to prove the stronger statement that for some orientation of $M$ and some $S^{1} \subset T$, the $S^{1}$-action has locally the same $S^{1}$-geometry as a suitable chosen $S^{1}$-action on one of the symmetric spaces $S^{8}$, $\mathbb{H} P^{2}$ or $\mathbb{C} P^{4}$ (that two $S^{1}$-manifolds have the same local $S^{1}$-geometry just means that there exists an equivariant orientation-preserving diffeomorphism between the normal bundles of the $S^{1}$-fixed point manifolds). Once this has been accomplished, one can glue the complements of the normal bundles together to get a new manifold $W$, with fixed point free $S^{1}$-action, that is bordant to the difference of $M$ and the symmetric space in question. As observed by Bott [1967], all Pontryagin numbers of a manifold with fixed point-free $S^{1}$-action vanish and hence $W$ is rationally zerobordant. Since the oriented bordism ring has no torsion in degree 8 , the manifold $M$ is bordant to the symmetric space in question.

This line of attack can be applied successfully at least if $\chi(M) \neq 5$. Details will appear elsewhere.

It is interesting to compare the results above with [Dessai and Tuschmann 2007], in which it is shown that there exists an infinite sequence of closed simply connected Riemannian 8-manifolds with nonnegative curvature and mutually distinct oriented bordism type.

\section{Acknowledgments}

Part of this work was carried out during a stay at the University of Pennsylvania, and I thank its mathematics department for its hospitality. I also thank Burkhard Wilking and Wolfgang Ziller for helpful discussions.

A particular step in the proof of Theorem 1.2 relies on a result about $\mathbb{Z} / 2 \mathbb{Z}$ cohomology $\mathbb{C} P^{4}$ 's. I am grateful to Mikiya Masuda for sharing this insight with me.

\section{References}

[Aloff and Wallach 1975] S. Aloff and N. R. Wallach, "An infinite family of distinct 7-manifolds admitting positively curved Riemannian structures", Bull. Amer. Math. Soc. 81 (1975), 93-97. MR 51 \#6851 Zbl 0362.53033

[Atiyah and Bott 1968] M. F. Atiyah and R. Bott, "A Lefschetz fixed point formula for elliptic complexes, II: Applications”, Ann. of Math. (2) 88 (1968), 451-491. MR 38 \#731

[Atiyah and Hirzebruch 1970] M. Atiyah and F. Hirzebruch, "Spin-manifolds and group actions", pp. 18-28 in Essays on Topology and Related Topics (Mémoires dédiés à Georges de Rham), edited by A. Haefliger and R. Narasimhan, Springer, New York, 1970. MR 43 \#4064 Zbl 0193.52401

[Atiyah and Singer 1968] M. F. Atiyah and I. M. Singer, "The index of elliptic operators, III", Ann. of Math. (2) 87 (1968), 546-604. MR 38 \#5245 Zbl 0164.24301

[Bott 1967] R. Bott, "Vector fields and characteristic numbers", Michigan Math. J. 14 (1967), 231244. MR 35 \#2297 Zbl 0145.43801 
[Bott and Taubes 1989] R. Bott and C. Taubes, "On the rigidity theorems of Witten", J. Amer. Math. Soc. 2:1 (1989), 137-186. MR 89k:58270

[Bredon 1972] G. E. Bredon, Introduction to compact transformation groups, Pure and Applied Mathematics 46, Academic Press, New York, 1972. MR 54 \#1265 Zbl 0246.57017

[Cheeger 1973] J. Cheeger, "Some examples of manifolds of nonnegative curvature", J. Differential Geometry 8 (1973), 623-628. MR 49 \#6085 Zbl 0281.53040

[Dessai and Tuschmann 2007] A. Dessai and W. Tuschmann, "Nonnegative curvature and cobordism type", Math. Z. 257:1 (2007), 7-12. MR 2008d:53037 Zbl 1127.53031

[Dessai and Wilking 2004] A. Dessai and B. Wilking, "Torus actions on homotopy complex projective spaces", Math. Z. 247:3 (2004), 505-511. MR 2006c:57033 Zbl 1068.57034

[Eells and Kuiper 1962] J. Eells, Jr. and N. H. Kuiper, "Manifolds which are like projective planes", Inst. Hautes Études Sci. Publ. Math. 14 (1962), 5-46. MR 26 \#3075 Zbl 0109.15701

[Eschenburg 1982] J.-H. Eschenburg, "New examples of manifolds with strictly positive curvature", Invent. Math. 66:3 (1982), 469-480. MR 83i:53061 Zbl 0484.53031

[Eschenburg 1992] J.-H. Eschenburg, "Cohomology of biquotients", Manuscr. Math. 75:2 (1992), 151-166. MR 93e:57070 Zbl 0769.53029

[Fang and Rong 2005] F. Fang and X. Rong, "Homeomorphism classification of positively curved manifolds with almost maximal symmetry rank", Math. Ann. 332:1 (2005), 81-101. MR 2005m: 53054 Zbl 1068.53022

[Frankel 1961] T. Frankel, "Manifolds with positive curvature”, Pacific J. Math. 11 (1961), 165-174. MR 23 \#A600 Zbl 0107.39002

[Frankel 1966] T. Frankel, "On the fundamental group of a compact minimal submanifold", Ann. of Math. (2) 83 (1966), 68-73. MR 32 \#4637

[Freedman 1982] M. H. Freedman, "The topology of four-dimensional manifolds", J. Differential Geom. 17:3 (1982), 357-453. MR 84b:57006 Zbl 0528.57011

[Friedlander and Halperin 1979] J. B. Friedlander and S. Halperin, "An arithmetic characterization of the rational homotopy groups of certain spaces", Invent. Math. 53:2 (1979), 117-133. MR 81f:55006b Zbl 0396.55010

[Gromov 1981] M. Gromov, "Curvature, diameter and Betti numbers", Comment. Math. Helv. 56:2 (1981), 179-195. MR 82k:53062 Zbl 0467.53021

[Grove and Halperin 1982] K. Grove and S. Halperin, "Contributions of rational homotopy theory to global problems in geometry", Inst. Hautes Études Sci. Publ. Math. 56 (1982), 171-177 (1983). MR 84b:58030 Zbl 0508.55013

[Grove and Searle 1994] K. Grove and C. Searle, "Positively curved manifolds with maximal symmetry-rank”, J. Pure Appl. Algebra 91:1-3 (1994), 137-142. MR 95i:53040 Zbl 0793.53040

[Grove and Ziller 2000] K. Grove and W. Ziller, "Curvature and symmetry of Milnor spheres", Ann. of Math. (2) 152:1 (2000), 331-367. MR 2001i:53047 Zbl 0991.53016

[Grove and Ziller 2008] K. Grove and W. Ziller, "Lifting group actions and nonnegative curvature", preprint, 2008. arXiv 0801.0767

[Grove et al. 2008] K. Grove, L. Verdiani, and W. Ziller, "A positively curved manifold homeomorphic to $T_{1} S^{4}$,, preprint, version 2, 2008. arXiv 0809.2304v2

[Halperin 1977] S. Halperin, "Finiteness in the minimal models of Sullivan", Trans. Amer. Math. Soc. 230 (1977), 173-199. MR 57 \#1493 Zbl 0364.55014

[Hamilton 1982] R. S. Hamilton, "Three-manifolds with positive Ricci curvature", J. Differential Geom. 17:2 (1982), 255-306. MR 84a:53050 Zbl 0504.53034 
[Hirzebruch 1968] F. Hirzebruch, "Involutionen auf Mannigfaltigkeiten", pp. 148-166 in Proc. Conf. on Transformation Groups (New Orleans, LA, 1967), edited by P. S. Mostert, Springer, New York, 1968. MR 40 \#3578 Zbl 0177.26401

[Hirzebruch et al. 1992] F. Hirzebruch, T. Berger, and R. Jung, Manifolds and modular forms, Aspects of Mathematics E20, Friedr. Vieweg, Braunschweig, 1992. MR 94d:57001 Zbl 0767.57014

[Hsiang 1975] W.-Y. Hsiang, Cohomology theory of topological transformation groups, Ergebnisse der Mathematik und ihrer Grenzgebiete 85, Springer, New York, 1975. MR 54 \#11363 Zbl 0429. 57011

[Hsiang and Kleiner 1989] W.-Y. Hsiang and B. Kleiner, "On the topology of positively curved 4manifolds with symmetry”, J. Differential Geom. 29:3 (1989), 615-621. MR 90e:53053 Zbl 0674. 53047

[James 1985] D. M. James, "Smooth $S^{1}$ actions on homotopy $\mathbf{C P}^{4}$ 's", Michigan Math. J. 32:3 (1985), 259-266. MR 87c:57031 Zbl 0602.57026

[Kapovitch 2002] V. Kapovitch, "A note on rational homotopy of biquotients", preprint, 2002, Available at http://www.math.toronto.edu/vtk/biquotient.pdf.

[Kapovitch and Ziller 2004] V. Kapovitch and W. Ziller, "Biquotients with singly generated rational cohomology”, Geom. Dedicata 104 (2004), 149-160. MR 2005e:22014 Zbl 1063.53055

[Kim 2008] J. H. Kim, "On positively curved 4-manifolds with $S^{1}$-symmetry”, preprint, version 1, 2008. arXiv 0810.1232v1

[Kobayashi 1972] S. Kobayashi, Transformation groups in differential geometry, Ergebnisse der Mathematik und ihrer Grenzgebiete 70, Springer, New York, 1972. MR 50 \#8360 Zbl 0246.53031

[Kramer and Stolz 2007] L. Kramer and S. Stolz, "A diffeomorphism classification of manifolds which are like projective planes”, J. Differential Geom. 77:2 (2007), 177-188. MR 2008g:57032 Zbl 1133.57017

[Lichnerowicz 1963] A. Lichnerowicz, "Spineurs harmoniques", C. R. Acad. Sci. Paris 257 (1963), 7-9. MR 27 \#6218 Zbl 0136.18401

[Masuda 1981] M. Masuda, "On smooth $S^{1}$-actions on cohomology complex projective spaces: The case where the fixed point set consists of four connected components", J. Fac. Sci. Univ. Tokyo Sect. IA Math. 28:1 (1981), 127-167. MR 82i:57031 Zbl 0462.57019

[Milnor 1963] J. Milnor, "Spin structures on manifolds", Enseignement Math. (2) 9 (1963), 198203. MR 28 \#622 Zbl 0116.40403

[Milnor and Stasheff 1974] J. W. Milnor and J. D. Stasheff, Characteristic classes, Annals of Mathematics Studies 76, Princeton University Press, 1974. MR 55 \#13428 Zbl 0298.57008

[Perelman 2002] G. Perelman, "The entropy formula for the Ricci flow and its geometric applications", preprint, 2002. Zbl 1130.53001 arXiv math.DG/0211159

[Perelman 2003a] G. Perelman, "Finite extinction time for the solutions to the Ricci flow on certain three-manifolds", preprint, 2003. Zbl 1130.53003 arXiv math.DG/0307245

[Perelman 2003b] G. Perelman, "Ricci flow with surgery on three-manifolds", preprint, 2003. Zbl 1130.53002 arXiv math.DG/0303109

[Petersen and Wilhelm 2008] P. Petersen and F. Wilhelm, "An exotic sphere with positive sectional curvature", preprint, 2008. arXiv 0805.0812

[Petrie 1973] T. Petrie, "Torus actions on homotopy complex projective spaces", Invent. Math. 20 (1973), 139-146. MR 48 \#1254 Zbl 0262.57021

[Püttmann and Searle 2002] T. Püttmann and C. Searle, "The Hopf conjecture for manifolds with low cohomogeneity or high symmetry rank", Proc. Amer. Math. Soc. 130:1 (2002), 163-166. MR 2002e:53044 
[Rong 2002] X. Rong, "Positively curved manifolds with almost maximal symmetry rank", Geom. Dedicata 95 (2002), 157-182. MR 2003m:53058 Zbl 1032.53025

[Singhof 1993] W. Singhof, "On the topology of double coset manifolds", Math. Ann. 297:1 (1993), 133-146. MR 94k:57054 Zbl 0793.57019

[Smale 1961] S. Smale, "Generalized Poincaré's conjecture in dimensions greater than four", Ann. of Math. (2) 74 (1961), 391-406. MR 25 \#580 Zbl 0099.39202

[Smale 1962] S. Smale, "On the structure of manifolds", Amer. J. Math. 84 (1962), 387-399. MR 27 \#2991 Zbl 0109.41103

[Sullivan 1996] D. P. Sullivan, "Triangulating and smoothing homotopy equivalences and homeomorphisms", pp. 69-103 in The Hauptvermutung book, edited by A. A. Ranicki, $K$-Monogr. Math. 1, Kluwer, Dordrecht, 1996. MR 98c:57027 Zbl 0871.57021

[Synge 1936] J. Synge, “On the connectivity of spaces of positive curvature.", Q. J. Math., Oxf. Ser. 7 (1936), 316-320. Zbl 0015.41601

[Totaro 2002] B. Totaro, "Cheeger manifolds and the classification of biquotients", J. Differential Geom. 61:3 (2002), 397-451. MR 2004b:53075 Zbl 1071.53529

[Wall 1960] C. T. C. Wall, "Determination of the cobordism ring", Ann. of Math. (2) 72 (1960), 292-311. MR 22 \#11403 Zbl 0097.38801

[Weinstein 1968] A. Weinstein, “A fixed point theorem for positively curved manifolds", J. Math. Mech. 18 (1968), 149-153. MR 37 \#3478 Zbl 0159.51401

[Wilking 2003] B. Wilking, "Torus actions on manifolds of positive sectional curvature", Acta Math. 191:2 (2003), 259-297. MR 2005g:53063 Zbl 1062.53029

[Wilking 2007] B. Wilking, "Nonnegatively and positively curved manifolds", pp. 25-62 in Metric and Comparison Geometry, edited by J. Cheeger and K. Grove, Surv. Differ. Geom. 11, Int. Press, Somerville, MA, 2007. MR 2009e:53048 Zbl 1162.53026

[Ziller 2007] W. Ziller, "Examples of Riemannian manifolds with non-negative sectional curvature", pp. 63-102 in Metric and Comparison Geometry, edited by J. Cheeger and K. Grove, Surv. Differ. Geom. 11, Int. Press, Somerville, MA, 2007. MR 2009h:53080 Zbl 1153.53033

Received September 18, 2009. Revised February 10, 2010.

\author{
ANAND DESSAI \\ DÉPARTEMENT DE MATHÉMATIQUES \\ CHEMin du MusÉE 23 \\ FACULTÉ DES SCIENCES \\ Université de Fribourg, PÉRolles \\ 1700 FRIBOURG \\ SWITZERLAND \\ anand.dessai@unifr.ch \\ http://homeweb1.unifr.ch/dessaia/pub/
}




\title{
PACIFIC JOURNAL OF MATHEMATICS
}

\author{
http://www.pjmath.org \\ Founded in 1951 by \\ E. F. Beckenbach (1906-1982) and F. Wolf (1904-1989)
}

\section{EDITORS}

V. S. Varadarajan (Managing Editor)

Department of Mathematics

University of California

Los Angeles, CA 90095-1555

pacific@math.ucla.edu

Vyjayanthi Chari

Department of Mathematics

University of California

Riverside, CA 92521-0135

chari@math.ucr.edu

Robert Finn

Department of Mathematics Stanford University

Stanford, CA 94305-2125

finn@math.stanford.edu

Kefeng Liu

Department of Mathematics

University of California

Los Angeles, CA 90095-1555

liu@math.ucla.edu
Darren Long

Department of Mathematics

University of California

Santa Barbara, CA 93106-3080

long@math.ucsb.edu

Jiang-Hua Lu

Department of Mathematics

The University of Hong Kong

Pokfulam Rd., Hong Kong jhlu@maths.hku.hk

Alexander Merkurjev

Department of Mathematics

University of California

Los Angeles, CA 90095-1555

merkurev@math.ucla.edu
Sorin Popa

Department of Mathematics University of California

Los Angeles, CA 90095-1555 popa@math.ucla.edu

Jie Qing

Department of Mathematics

University of California

Santa Cruz, CA 95064

qing@cats.ucsc.edu

Jonathan Rogawski

Department of Mathematics

University of California

Los Angeles, CA 90095-1555

jonr@math.ucla.edu

\section{PRODUCTION}

pacific@math.berkeley.edu

\begin{abstract}
Silvio Levy, Scientific Editor Matthew Cargo, Senior Production Editor
\end{abstract}
ACADEMIA SINICA, TAIPEI

CALIFORNIA INST. OF TECHNOLOGY

INST. DE MATEMÁTICA PURA E APLICADA

KEIO UNIVERSITY

MATH. SCIENCES RESEARCH INSTITUTE

NEW MEXICO STATE UNIV.

OREGON STATE UNIV.

\section{SUPPORTING INSTITUTIONS}

STANFORD UNIVERSITY
UNIV. OF BRITISH COLUMBIA
UNIV. OF CALIFORNIA, BERKELEY
UNIV. OF CALIFORNIA, DAVIS
UNIV. OF CALIFORNIA, LOS ANGELES
UNIV. OF CALIFORNIA, RIVERSIDE
UNIV. OF CALIFORNIA, SAN DIEGO
UNIV. OF CALIF., SANTA BARBARA

UNIV. OF CALIF., SANTA CRUZ

UNIV. OF MONTANA

UNIV. OF OREGON

UNIV. OF SOUTHERN CALIFORNIA

UNIV. OF UTAH

UNIV. OF WASHINGTON

WASHINGTON STATE UNIVERSITY

These supporting institutions contribute to the cost of publication of this Journal, but they are not owners or publishers and have no responsibility for its contents or policies.

See inside back cover or www.pjmath.org for submission instructions.

The subscription price for 2011 is US \$420/year for the electronic version, and \$485/year for print and electronic.

Subscriptions, requests for back issues from the last three years and changes of subscribers address should be sent to Pacific Journal of Mathematics, P.O. Box 4163, Berkeley, CA 94704-0163, U.S.A. Prior back issues are obtainable from Periodicals Service Company, 11 Main Street, Germantown, NY 12526-5635. The Pacific Journal of Mathematics is indexed by Mathematical Reviews, Zentralblatt MATH, PASCAL CNRS Index, Referativnyi Zhurnal, Current Mathematical Publications and the Science Citation Index.

The Pacific Journal of Mathematics (ISSN 0030-8730) at the University of California, c/o Department of Mathematics, 969 Evans Hall, Berkeley, CA 94720-3840, is published monthly except July and August. Periodical rate postage paid at Berkeley, CA 94704, and additional mailing offices. POSTMASTER: send address changes to Pacific Journal of Mathematics, P.O. Box 4163, Berkeley, CA 94704-0163.

PJM peer review and production are managed by EditFLOW ${ }^{\mathrm{TM}}$ from Mathematical Sciences Publishers.

PUBLISHED BY PACIFIC JOURNAL OF MATHEMATICS

at the University of California, Berkeley 94720-3840

A NON-PROFIT CORPORATION

Typeset in LATEX

Copyright $(02011$ by Pacific Journal of Mathematics 


\section{PACIFIC JOURNAL OF MATHEMATICS}

Volume $249 \quad$ No. $1 \quad$ January 2011

Metabelian SL $(n, \mathbb{C})$ representations of knot groups, II: Fixed points

HANS U. BODEN and STEFAN FRIEDL

Lewis-Zagier correspondence for higher-order forms

ANTON DEITMAR

Topology of positively curved 8-dimensional manifolds with symmetry

ANAND DESSAI

Strong Kähler with torsion structures from almost contact manifolds

MARISA FERnÁNDEZ, ANNA FINO, LUIS UGARTE and RAQUEL

VILLACAMPA

Connections between Floer-type invariants and Morse-type invariants of Legendrian knots

MICHAEL B. HENRY

A functional calculus for unbounded generalized scalar operators on Banach spaces

DRAGOLJUB KEČKIĆ and ĐORĐE KRTINIĆ

Geometric formality of homogeneous spaces and of biquotients

D. KoTSCHICK and S. TERZIĆ

Positive solutions for a nonlinear third order multipoint boundary value problem

Yang LiU, Zhang Weiguo, Liu Xiping, Shen Chunfang and Chen HUA

The braid group surjects onto $G_{2}$ tensor space

SCOTT MORRISON

Analogues of the Wiener Tauberian and Schwartz theorems for radial functions on symmetric spaces

E. K. NARAyAnAn and Alladi Sitaram

Semidirect products of representations up to homotopy

Yunhe SHENG and Chenchang ZHU

Homology sequence and excision theorem for Euler class group

YONG YANG 